Rev. Elev. Méd. vét. Pays trop., 1971, 24 (2) : 233-38

\title{
Note sur la contamination bactérienne des crevettes pêchées à Madagascar
}

\author{
par J. BLANCOU et F. BLANC \\ (avec la collaboration technique de D. RAKOTOSON)
}

\section{RESUME}

Les techniques de pêche, stockage, commercialisation des crevettes à Madagascar sont décrites. Les résultats des analyses bactériologiques et biochimiques de leur contamination sont exposés et des hormes sont proposées en fonction de l'examen organoleptique de certains échantillons.

\section{INTRODUCTION}

Depuis 1966 se développe sur la Côte Ouest de Madagascar une pêche industrielle de la crevette. La production annuelle atteint 1.500 tonnes, dont la majeure partie est exportée à l'état congelé au Japon. Il s'agit de "Penaeides" dont le cycle biologique se déroule tout près des côtes, de la mangrove aux fonds vaseux ou sabloneux. Elles se rencontrent surtout à l'embouchure des cours d'eaux chargés de matières organiques.

\section{Méthode de pêche}

Pêche artisanale et pêche industrielle sont également pratiquées sur la Côte Ouest. Seule cette dernière sera envisagée dans la présente étude. Dans la méthode de pêche par chalutage chaque bateau traine sur le fond marin deux chaluts, relevés, vidés et remis périodiquement à l'eau.

\section{Méthode de conditionnement des crevettes pêchées}

Les crevettes déchargées sur le pont préalablement lavé au jet d'eau sont séparées des autres prises : crabes, poissons, éponges. Elles sont ensuite jetées dans des bacs métalliques grillagés et lavées au jet d'eau, puis disposées dans des plateaux d'aluminium, mélangées avec de la glace ( $1 \mathrm{~kg}$ de crevettes pour $2,5 \mathrm{~kg}$ de glace). Ces plateaux sont placés en cale isotherme jusqu'au débarquement.

Celui-ci s'effectue à quai, à Majunga. Les bacs sortis de la cale sont aussitôt entreposés dans des chambres de stockage (à $\left.+4^{\circ}\right)$ où ils séjournent de 1 à 48 heures. Les crevettes sont ensuite lavées, triées par taille, calibrées et placées en cartons paraffinés. L'opération se déroule dans un hangar clos, non isolé au point de vue thermique (température intérieure : 20 à $30^{\prime \prime}$ ). Les cartons paraffinés sont aussitôt refroidis à $-40^{\circ}$ durant 25 minutes puis conservés à $-25^{\circ}$ durant 30 à 45 jours au plus.

\section{Méthode d'étude}

Nous nous sommes limités dans cette étude aux crevettes de pêche industrielle puisqu'il s'agit de la pêche la plus importante d'un point de vue économique, dont le contrôle à l'exportation doit être régulièrement effectué. Nous nous sommes également limités à l'étude de l'évolution quantitative de la flore bactérienne, et à ses conséquences.

L'identification des bactéries hôtes normaux du crustacé a fait l'objet d'une étude séparée, sur crevettes vivantes. 
L'appréciation de la dégradation du crustacé depuis sa pêche jusqu'à sa consommation peut être faite selon deux méthodes $(4,5,6)$ :

- étude de la modification qualitative et quantitative de sa population bactérienne;

- étude indirecte des modifications biochimiques entraînées par cette variation bactérienne.

Nous avons simultanément utilisé ces deux méthodes.

\section{MATERIEL}

Les crevettes ont été étudiées à tous les stades de la pêche et de la conservation. Un total de 272 prélèvements a été analysé. Afin de simplifier l'exposé des résultats, nous avons distingué quatre catégories de prélèvements:

1. Crevettes vivantes.

2. Crevettes à la sortie des cales du chalutier. (où elles ont déjà effectué un séjour de 1 à 6 jours).

3. Crevettes après triage et conservation au surgélateur (où elles ont effectué un séjour de 30 à 45 jours).

4. Crevettes décongelées et conservées soit à $+4^{\circ}$ soit à la température ambiante.

\section{METHODES}

Les crevettes destinées aux analyses subissent le traitement suivant :

- Prélèvement de 50 grammes de crevettes.

- Broyage 5 minutes dans un appareil type «Mixer » refroidi, stérilisé, en présence de $150 \mathrm{ml}$ de «diluant universel » (1) de formule suivante :

Tryptone DIFCO . . . . $1 \mathrm{~g}$

Chlorure de Sodium . . . $\quad 8,5 \mathrm{~g}$

Eau distillée . . . . . $1.000 \mathrm{ml}$

(pH 7 stérilisé 20 minutes à $\left.120^{\circ}\right)$.

On admet que un millilitre de la suspension résultante représente 0,25 grammes de crevettes : c'est à partir d'elle que son effectuées les dilutions en progression décimale du produit. Egalement à partir de cette suspension sont réalisées les mesures du $\mathrm{pH}$ et le dosage de l'azote basique volatil total. Un autre prélèvement doit être effectué à part pour la mise en évidence de l'indol et de la cadavérine.

\section{METHODES BACTERIOLOGIQUES}

Ces méthodes visent à dénombrer ou définir la population bactérienne de la crevette au cours des différentes étapes de sa conservation $(2,3)$.

\section{Numération des bactéries aérobies et aéro-anaérobies}

La numération se fait à partir des dilutions, en progression logarithmique, de la suspension initiale. Elles sont ensemencées sous le volume de $1 \mathrm{ml}$ dans la masse d'une gélose nutritive ordinaire. La couche de gélose une fois refroidie est recouverte d'une mince pellicule de gélose pour éviter l'envahissement en nappe par les bactéries. On admet que une colonie représente une bactérie.

\section{Numération des bactéries anaérobies strictes}

Les différentes dilutions sont ensemencées en gélose "Viande-Foie " en tubes de $8 / 180 \mathrm{~mm}$. Les colonies d'anaérobies strictes sont dénombrées dans la zone située à $2 \mathrm{~cm}$ au-dessous de la surface.

\section{Numération des bactéries protéolytiques}

Bactéries productrices d'hydrogène sulfureux: ensemencement sur gélose au sous-acétate de plomb, la présence de ces bactéries se traduit par un précipité noir de sulfure de plomb.

Bactéries productrices d'Indol: ensemencement en eau peptonée, l'indol y est recherché après 48 heures par addition de $0,5 \mathrm{ml}$ de réactif d'EHRLICH KOVACS.

Bactéries gélatinolytiques: ensemencement en eau peptonée. La présence de bactéries protéolytiques y est recherchée après 48 heures par immersion d'une pellicule de film noir de $5 \times 10 \mathrm{~mm}$, selon la technique de LE MINOR et PIECHAUD (1).

\section{Numération des bactéries de contamination entérique}

Les différentes dilutions sont ensemencées sur les milieux suivants, les résultats étant lus après 48 heures d'incubation à $37^{\circ}$ :

Escherichia coli : sur le milieu de TEAGUELEVINE. Les bactéries fermentant le lactose, 
dont les colonies sont violettes, sont assimilées à des $E$. coli. Cette approximation a été contrôlée sur 100 colonies dont l'identification complète a été poursuivie : elle est vérifiée dans 85 p. 100 des cas.

Streptocoques fécaux: sur le milieu de SLANETZ et BARTLEY-DIFCO. Ce milieu n'est pas totalement inhibiteur pour les autres bactéries, en particulier les microcoques. Cependant dans plus de 80 p. 100 des cas, les petites colonies rouges ou marrons, possédant une catalase, isolées sur ce milieu ont été identifiées par leurs caractères biochimiques comme des Streptocoques fécaux: Streptococcus foecium et faocalis dans la majorité des cas. (7)

Clostridies sulfito-réductrices: sur le milieu de WILSON et BLAIR (gélose viande foie additionnée de sulfite de sodium et d'alun de fer ammoniacal).

La gélose est chauffée 10 minutes à $80^{\circ}$ afin de sélectionner les Clostridium thermo-résistants dont les colonies sont entourées d'un dépôt noir de sulfure de fer. Nous avons poursuivi l'identification de 37 colonies de ce type: il s'agissait dans 22 cas de Welchia perfringens.

\section{Numération des staphylocoques}

Les différentes dilutions sont ensemencées sur le milieu de CHAPMANN. Sur ce milieu 95 p. 100 des colonies possédant une catalase sont bien des staphylocoques. Les colonies contrôlées sont ensemencées sur bouillon spécial pour la recherche de la staphylo-coagulase. Les souches étudiées possédaient une coagulase dans 65 p. 100 des cas (étude sur 64 souches).

\section{METHODES BIOCHIMIQUES}

Nous avons utilisé exactement les méthodes décrites par J. PANTALEON et R. ROSSET pour le contrôle de la salubrité des poissons et coquillages (4). On trouvera une description détaillée des techniques employées dans leur publication.

1. Mesure $d u p H$ : elle est effectuée sur la suspension initiale, au papier indicateur ou au pHmètre électrique.

2. Recherche de la cadavérine: le prélèvement est broyé, alcalinisé, puis subit une extrac- tion chloroformique. La cadavérine est caractérisée par la ninhydrine.

3. Recherche de l'indol: l'indol est caractérisé, dans l'extrait chloroformique précédent, par le réactif d'EHRLICH.

4. Dosage de l'ammoniac et des amines volatiles totales: l'opération est effectuée dans un ballon relié à un réfrigérant descendant, selon la technique décrite par les auteurs. Le résultat est exprimé en milligrammes d'ammoniac.

\section{RESULTATS}

Les résultats sont exposés dans le tableau $n^{*}$ I. Nous n'y faisons figurer ni les résultats de la recherche de la cadavérine (qui ont toujours été négatifs) ni ceux de la recherche de l'indol (trop rarement positifs pour être significatifs).

Pour l'étude bactériologique le nombre de colonies bactériennes est indiqué par l'exposant logarithmique moyen des dilutions ayant donné naissance à des colonies en gélose.

Ces résultats nous ont paru plus clairement exprimés par des graphiques correspondants. Nous n'en avons, volontairement fait figurer que quatre. Ce sont ceux qui, par leur progression régulière, sont le plus indiqués comme critère d'appréciation de l'état de dégradation des crustacés.

\section{CONCLUSION - DISCUSSION}

Le but de ce travail était de distinguer les épreuves qui reflètent le plus fidèlement possible la dégradation progressive des crevettes. D'après le tableau $\mathrm{n}^{\circ}$ I et les graphiques, il semble que les techniques les mieux adaptées à cet effet soient les suivantes, par ordre d'efficacité.

1. Mesure de l'A.B.V.T.

2. Numération des streptocoques fécaux.

3. Mesure du $\mathrm{pH}$.

4. Numération des staphylocoques.

5. Autres méthodes.

Nous ne retenons, pour des raisons pratiques, que les épreuves qui ont l'avantage de 
TABLEAU $\mathrm{N}^{\circ} \mathrm{I}$

\begin{tabular}{|c|c|c|c|c|c|c|c|c|c|c|c|c|}
\hline \multirow{2}{*}{\multicolumn{2}{|c|}{ Prélèvement }} & \multirow[b]{2}{*}{$\mathrm{pH}$} & \multirow[b]{2}{*}{$\mathrm{ABVT} *$} & \multicolumn{5}{|c|}{ Bactéries } & \multirow[b]{2}{*}{ 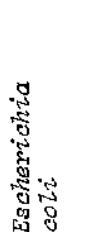 } & \multirow[b]{2}{*}{ 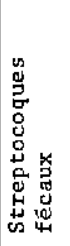 } & \multirow[b]{2}{*}{$\begin{array}{l}0 \\
0 \\
0 \\
0 \\
0 \\
0 \\
0 \\
0 \\
0 \\
0 \\
0 \\
0 \\
0 \\
0\end{array}$} & \multirow{2}{*}{ 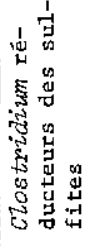 } \\
\hline & & & & 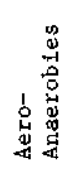 & 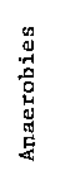 & 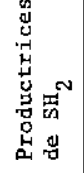 & 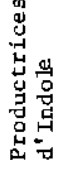 & 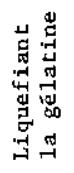 & & & & \\
\hline \multicolumn{2}{|c|}{ Crevettes vivantes } & 7,2 & - & 4,1 & 0,8 & 0,1 & 1,1 & 0,1 & 0 & 0,2 & 0,2 & 0,1 \\
\hline \multicolumn{2}{|c|}{$\begin{array}{l}\text { Crevettes à la sortie } \\
\text { de la cale }\end{array}$} & 7,2 & 30 & 4,5 & $0, B$ & 0,1 & 1,2 & 0,1 & 0 & 0,6 & 0,3 & 0,1 \\
\hline \multicolumn{2}{|c|}{$\begin{array}{l}\text { Grevettes trlées et } \\
\text { congelées }\end{array}$} & 7,3 & 32 & 4,5 & 0,7 & 0,4 & 1,4 & 0 & 0 & 0,8 & 0,7 & 0 \\
\hline \multirow{4}{*}{$\begin{array}{l}\text { Crevettes } \\
\text { prêcêdentes } \\
\text { conservées } \\
\text { à }+4^{\circ}\end{array}$} & $\begin{array}{l}\text { après } \\
24 \mathrm{~h} \text {. }\end{array}$ & 7,4 & 35,5 & 4,8 & 3,1 & 0,5 & 1,1 & 1,3 & 0 & 1,0 & 2,0 & 0,4 \\
\hline & $\begin{array}{l}\text { après } \\
48 \mathrm{~h} .\end{array}$ & 8,2 & 42,4 & 5,1 & 3,4 & 0,8 & 1,3 & 1,7 & 0 & 1,1 & 2,1 & 0,6 \\
\hline & $\begin{array}{l}\text { après } \\
72 \mathrm{~h} .\end{array}$ & 8,5 & 53,6 & 5,5 & 3,7 & 1,1 & 1,4 & 2,4 & 0 & 1,5 & 3,3 & 0,8 \\
\hline & $\begin{array}{c}\text { après } \\
\text { plus } \\
\text { de } 72 \mathrm{~h}\end{array}$ & 9,0 & $88, \mathrm{~B}$ & 6,1 & 4,2 & 1,2 & 1,9 & 2,7 & 0 & 1,7 & 3,4 & 1,0 \\
\hline \multirow{3}{*}{$\begin{array}{l}\text { Crevettes } \\
\text { précedentes } \\
\text { conservées } \\
\text { température } \\
\text { amb1ante } \\
\left(+16 \text { à }+28^{\circ}\right)\end{array}$} & $\begin{array}{l}\text { après } \\
24 \mathrm{~h} .\end{array}$ & 7,8 & 58,5 & 6,07 & 3,9 & 3,0 & 2,7 & 2,6 & 0,6 & 1,2 & 3,7 & 0,6 \\
\hline & $\begin{array}{l}\text { après } \\
48 \mathrm{~h} .\end{array}$ & 8,9 & 125,6 & 7,2 & 5,4 & 3,4 & 3,3 & 4 & 1,0 & 1,7 & 4,3 & 0,7 \\
\hline & $\begin{array}{l}\text { après } \\
72 \mathrm{~h} .\end{array}$ & 9,1 & 196,5 & 7,6 & 6,1 & 4,5 & 4,4 & 4,3 & 1,1 & 3,1 & 5,0 & 1,1 \\
\hline
\end{tabular}

* Azote basique volatil total.

pouvoir se pratiquer dans des conditions de stérilité très relatives, les seules épreuves bactériologiques utilisant un milieu sélectif, dont la contamination au cours des manipulations est très malaisée.

Restait à déterminer, en fonction des épreuves choisies, les seuils au-delà desquels la consommation des crevettes est désagréable, voire impossible. Pour cela nous avons cons- titué des «jurys de dégustation» de 4 personnes. Après dégustation des différents lots soumis à leur appréciation les lots ont été classés, et les résultats des 4 épreuves choisies (déterminés d'après un échantillonnage effectué sur le lot) indiquées pour chaque classement.

D'après le tableau $\mathrm{n}^{\circ} 2$, il est aisé de déterminer les seuils qui pourraient être choisis pour définir les qualités d'un lot de crevettes.

\section{LEGENDE DES GRAPHIQUES}

1. Evolution de la quantité d'A.B.V.T. (en mg) par gramme de crevettes à $+25^{\circ}$ ou à $4^{0}$.

2. Evolution du pH à $+25^{\circ}$ ou à +40 .

3. Evolution du nombre de streptocoques fécaux par gramme de crevettes à $+25^{\circ}$ ou à $+4^{\circ}$.

4. Evolution du nombre de staphylocoques par gramme de crevettes à $+25^{\prime \prime}$ ou à $+4^{\text {n }}$. 


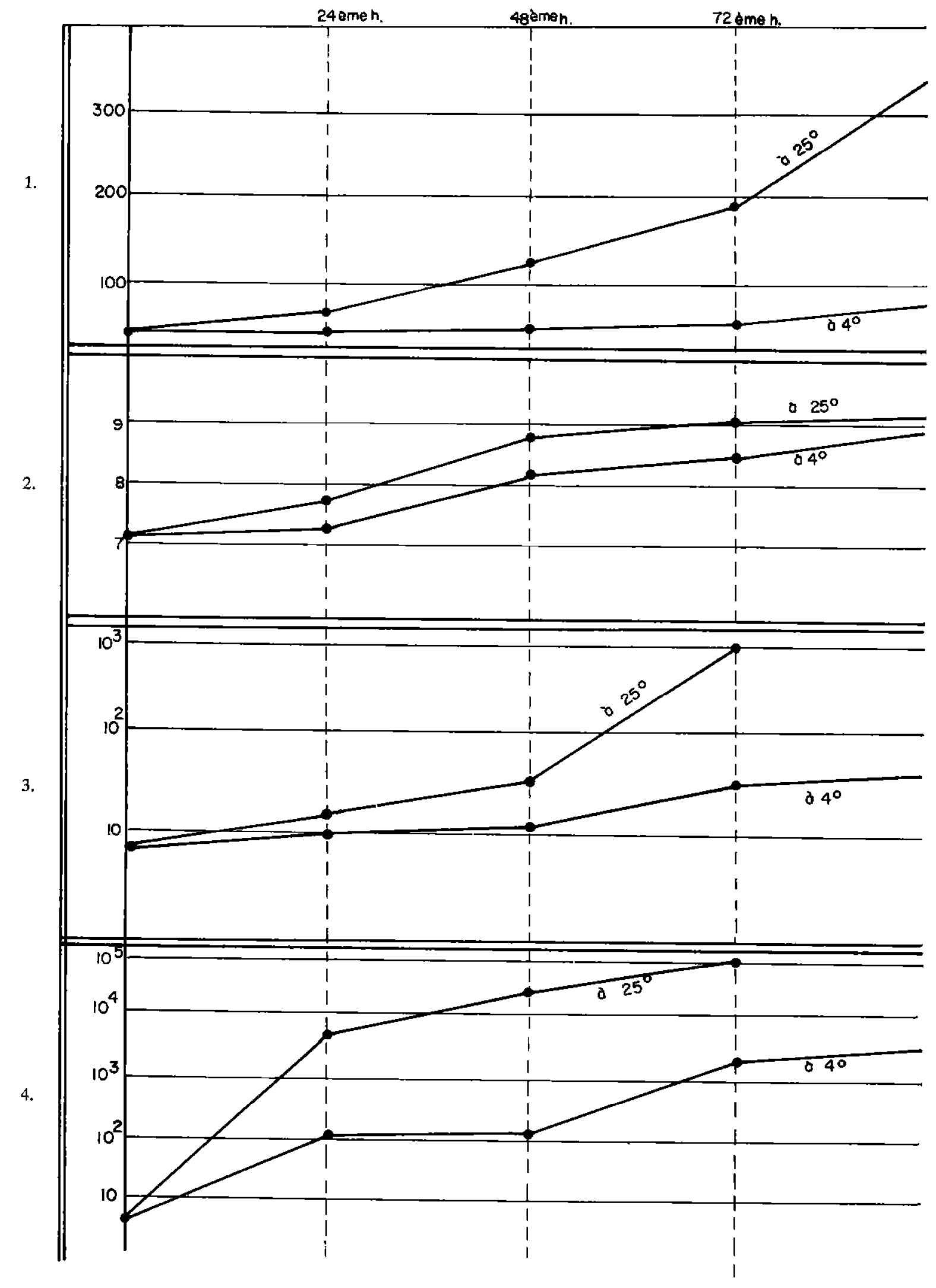


TABLEAU $\mathrm{N}^{\circ} \mathrm{II}$

\begin{tabular}{|c|c|c|c|c|}
\hline \multirow[b]{2}{*}{ Classement } & \multicolumn{4}{|c|}{ E preu ve } \\
\hline & A.B.V.T. * & $\begin{array}{l}\text { Quantité de } \\
\text { Streptocoques-fêcaux }\end{array}$ & $\mathrm{pH}$ & $\begin{array}{c}\text { Quantité de } \\
\text { Staphylocoques }\end{array}$ \\
\hline Très bon & 30 à 35 & 0,5 à 1 & 7 à 7,5 & 0,5 à $0, B$ \\
\hline Bon & 35 à 45 & $0, B$ à 1 & 7,5 à 8 & 0,8 à 2 \\
\hline $\begin{array}{l}\text { Dësagréable tnais } \\
\text { cons ommable }\end{array}$ & 45 à 60 & 1 à 1,5 & $B \quad$ a 8,5 & 2 à 3 \\
\hline Incon sommab le & $\underset{60}{\text { au-dẹà de }}$ & $\begin{array}{c}\text { au-delà de } \\
1,5\end{array}$ & $\begin{array}{l}\underset{8,5}{\text { au-delà de }} \\
\qquad, 5\end{array}$ & $\underset{3}{\operatorname{au}-d e 1 a ̃ ~ d e ~}$ \\
\hline
\end{tabular}

* Azote basique volatil total.

\section{SUMMARY}

\section{Note about bacterial contamination of shrimps fished in Madagascar}

Processes of capture, stockage, and trade of shrimps in Madagascar are described. Results of bacteriological and biochemical analysis of their contamination are given, and standards are suggested according to sensory evaluation of some samples.

\section{RESUMEN}

\section{Nota sobre la contaminación bacteriana de las gambas pescadas} en Madagascar

Se describen las téćnicas de pesca, almacenamiento, comercialización de las gambas en Madagascar. Se exponen los resultados de las analisis bacteriológicas y bioquimicas de su contaminación y se proponen normas según el examen organoleptico de ciertas muestras.

\section{BIBLIOGRAPHIE}

1. BUTTLAUX (R.), BEERENS (H.) et TACQUET (A.), "Manuel des techniques bactériologiques», Paris, Ed. Médicales Flammarion, 1969.

2. F.A.O., «The Technology of fish utilisation», FAO international Symposium, May 1964.

3. LEBERT (F.), « Chronique de bactériologie alimentaire. Normes et techniques pour le contrôle bactériologique des produits alimentaires destinés aux armées $x$, Rev. Serv. biol. vét. Armées, 1962, (2) : 11.

4. PANTALEON (J.) et ROSSET (R.), «Contrôle de la qualité et de la salubrité du poisson et des coquillages - Epreuves de laboratoire simples et rapides », Rev. Conserve, 1963, $7: 331-62$.

5. PENSO (G.), "Les produits de la pêche », Paris, Vigot Frères, 1953.

6. SOUDAN (F.), « La conservation par le froid des poissons, crustacés et mollusques », Paris, J. Baillière et Fils, 1965. (Encyclopédie du Froid.)

7. SUREAU (P.) et SERRES (H.), "Note préliminaire sur l'utilisation du milieu de SLANETZ et BARTLEY pour la recherche et la numération des streptocoques fécaux dans les laits, Arch. Inst. Pasteur Maroc, 1959, 27 : 23-31. 